\section{MUJER Y TEOLOGÍA: LA CUESTIÓN DE LA IMAGEN DE DIOS}

\author{
Blanca Castilla de Cortázar \\ Real Academia de Doctores de España \\ castilla@blancascor@gmail.com
}

Cómo citar este artículo/Citation: Castilla de Cortázar, B. (2016). "Mujer y Teología: la cuestión de la imagen de Dios". Arbor, 192 (778): a302. doi: http://dx.doi.org/10.3989/ arbor.2016.778n2005

Recibido: 08 septiembre 2015. Aceptado: 14 septiembre 2015.

RESUMEN: La cuestión de la imagen de Dios, afirmada en el libro del Génesis, es un tema teológico con amplio recorrido, acerca del cual se ha avanzado en las últimas décadas, en relación con la teología de la mujer, tarea aún pendiente. En el presente trabajo, tras un breve iter en torno al progreso de esta cuestión, nos detendremos a analizar la novedosa propuesta de Karol Wojtyla en su obra magna: La teología del cuerpo, analizando su método, sus puntos de partida y sus principales consecuencias, para concluir señalando las tareas pendientes.

PALABRAS CLAVE: Teología de la mujer; imagen de Dios; sexualidad; andrógino; reciprocidad; complementariedad; esponsalidad; dualidad; transcendentalidad.

\section{WOMEN AND THEOLOGY. THE QUESTION OF THE IMAGE OF GOD}

Copyright: () 2016 CSIC. Este es un artículo de acceso abierto distribuido bajo los términos de la licencia Creative Commons Attribution (CC BY) España 3.0.

ABSTRACT: The question of the image of God, described in the book of Genesis, is a long-standing theological issue. Although there has been some progress in this regard in recent decades, particularly in relation to the theology of women, there is still work to be done. In this paper, after a brief iter on the progress on this matter, we take a closer look at Karol Wojtyla's novel proposal in his magnum opus: The theology of the body, analyzing his method, starting points and main consequences. We conclude by pointing out the tasks ahead.

KEYWORDS: Theology of women; image of God; sexuality; androgyne; reciprocity; complementarity; spousal character; duality; transcendentality. 
En el primer libro de la Biblia, en dos versículos (Génesis 1, 26-27), se afirma por tres veces que el ser humano fue creado "a imagen de Dios". Sin embargo, el contenido y el significado de esa imagen, la base inmutable de toda la antropología cristiana, es uno de los temas teológicos de más amplio recorrido a lo largo de siglos de pensamiento. En ese dilatado iter se pueden distinguir cuatro pasos:

1. Solo el varón es imagen de Dios, Eva es derivada.

2. La imagen está en el alma y esta es asexuada.

3. Tanto el varón como la mujer en cuanto tales son imagen de Dios.

4. La plenitud de la imagen está en la "unidad de los dos", que viene a ser una imagen de la "unidad Trinitaria".

Como puede advertirse, en el centro de este proceso se dirime la cuestión de si la mujer es, y de qué modo, imagen de Dios. Los dos últimos pasos son aún recientes y están sin terminar de desarrollar y en ellos se encuentra la clave para el desarrollo de una teología sobre la mujer que aún es una tarea pendiente. $Y$ no solo por parte de la teología, sino también por falta de un avance paralelo en el ámbito de la antropología filosófica de la que, en cierto modo, la teología depende. Ciertamente, el conocimiento de la propia sexualidad y su llamada al amor, no son fáciles de tematizar y necesitan una investigación que reúna ciencia, antropología y ética; así como el concurso de la teología, ciencia que requiere a su vez los datos de las demás ciencias para profundizar y poder interpretar de un modo certero el sentido de los datos revelados, iluminando así el resto de los saberes. Como veremos, algunos de los déficits hoy superados de algunas opiniones teológicas se deben a las lagunas o errores científicos, que se barajaban en el momento en que se formularon dichas teorías.

En el presente trabajo, tras un breve iter en torno al progreso de esta cuestión nos detendremos a analizar la novedosa propuesta de Karol Wojtyla, analizando su método, sus puntos de partida y sus principales consecuencias, para concluir señalando las tareas pendientes.

\section{DIFICULTADES HERMENÉUTICAS}

Como es sabido, en el libro del Génesis se recogen dos relatos de la creación. En el primero de ellos, en el corto espacio de dos versículos (26-27) se afirma por tres veces que el hombre (varón y mujer) es creado a imagen de Dios. Inmediatamente después, en Génesis 2, se recoge otro relato de la creación de la primera pareja humana (el conocido como "de la costilla de Adán") que, con un lenguaje simbólico y metafórico y sin nombrarla directamente, desarrolla el contenido de dicha imagen. Sin embargo, sin reparar en que no puede haber contradicción con lo ya relatado en el primero, durante siglos ese segundo pasaje se ha interpretado literalmente, como si la mujer hubiera aparecido posteriormente en el tiempo; y apoyándose en una interpretación sesgada se ha querido encontrar en él la justificación a posturas subordinadas, afirmando por ejemplo, que en cierto sentido "el varón es principio y fin de la mujer, como Dios es principio y fin de toda criatura" (Tomás de Aquino, I, q. 93, a. 4, ad 1).

Esta interpretación "antigua" - como la ha denominado Juan Pablo II- ha estado frecuentemente condicionada por arcaicos mitos como el del andrógino de Platón (189c-193d), que han difuminado aspectos centrales del contenido del mensaje bíblico. Entre ellos no ha sido infrecuente considerar que la diversidad sexual proviene de un castigo divino que partió en dos una identidad originaria. La relación entre los sexos se planteó entonces como una búsqueda de la propia plenitud, en lugar de un don que se da a otro por amor. Esta influencia, con diversos matices, ha permanecido vigente hasta la actualidad.

Otro de los condicionamientos de la antropología diferencial varón-mujer ha sido los errores culturales provocados por el desconocimiento de la fisiología humana. Desde antiguo, como recoge Aristóteles (IVa.C./1994, p. 113 y p. 273), se pensó que solo el varón engendraba, siendo considerada la mujer únicamente como tierra fecunda en la que germina la semilla en ella sembrada. De ahí que desde el $\mathrm{s}$. $\mathrm{V}$ antes de Cristo se impusiera la imagen de la maternidad y la mujer como pasividad, frente al hombre-varón que representaba actividad. Ese error solo se disipó científicamente cuando a finales del siglo XIX se descubrió la fecundación (Gomis Blanco, 1991, p. 11). Más aún, la ciencia ha demostrado que en el origen de un nuevo ser humano la mujer aporta más que el varón, pues solo de ella procede el ADN mitocondrial contenido en el citoplasma del óvulo. Aun así, en pleno siglo XX antropológicamente esos prejuicios siguen sin haber sido superados y algunos tratados siguen manteniendo la supuesta pasividad femenina (Buytendijk, 1966).

En tercer lugar, habría que nombrar la influencia del platonismo plotiniano, según la cual la plenitud no es plural sino monolítica, y de ella se deriva el ideal de la autosuficiencia en lugar de la riqueza de la coope- 
ración. En esta línea se considera que solo el uno es supremo, por lo que el dos necesariamente ha de estar subordinado. De esta manera, la alteridad no ha conseguido ser reconocida como tal, siendo reducida a una copia deformada de un único modelo. Durante muchos siglos la mujer ha sido considerada como la copia deficiente del hombre-varón, que es quien encarna el prototipo humano. Tal visión, como puede advertirse, no hace justicia a la mujer ni al varón que permanece prisionero de una soledad que excluye toda verdadera relación recíproca.

En resumidas cuentas, la exégesis ha estado condicionada por los prejuicios, inherentes a la condición caída del hombre. Por esta razón, en las últimas décadas una de las principales tareas de la ciencia bíblica es discernir y separar el mensaje divino de los envoltorios culturales que le acompañan.

\section{ITER DE LA INVESTIGACIÓN SOBRE LA IMAGEN DE DIOS}

Haciendo un poco de historia, en la tradición judía se consideró que solo el varón era imagen de Dios, mientras que la mujer era derivada. Esto ha justificado la situación subordinada de la mujer en el mundo judío y musulmán en los que (sobre todo en este último) aún hoy se encuentra encerrada.

Con la época cristiana se abre una nueva era al ser conscientes de la dignidad de la mujer recuperada por Jesucristo. Todo el mundo quedó sorprendido al ver cómo trata el Mesías a las mujeres, cuya dignidad ha tenido siempre como icono la figura de la Virgen, la criatura más perfecta. También los textos apostólicos lo afirman con claridad: "Ya no hay judío ni griego, esclavo ni libre, varón ni mujer, porque todos sois uno en Cristo", afirma Pablo de Tarso en su carta a los Gálatas $(3,28)$. "En relación a lo "antiguo", esto es evidentemente "nuevo": es la novedad evangélica", afirma Juan Pablo II (1988, n. 24)

Por su parte, los Padres de la Iglesia y los pensadores cristianos a partir de Clemente de Alejandría y de san Agustín, que son los primeros en argumentar que la mujer es imagen de Dios, se esforzaron para desarrollar el contenido de la imagen de Dios, que se amplía para contener a ambos (varón y mujer). Esto supone que el Cristianismo ha puesto la primera piedra miliar inamovible en la dilucidación de esta cuestión, defendiendo de un modo indiscutible la igualdad entre ambos.

Pero la cuestión no es tan fácil porque junto con la igualdad es preciso articular la diferencia, cuestión que aún permanece pendiente, debido en parte a que tampoco está resuelta por las ciencias humanas. Siguiendo con el proceso de clarificación-que siempre avanza rectificando errores-, dentro del pensamiento cristiano se dio un paso adelante con la tesis de que la imagen de Dios pertenece al alma y que esta es asexuada. Con respecto a la posición anterior este desarrollo supone un avance significativo, aunque no exento de obstáculos. Señalemos algunos.

El primer escollo se encuentra en los propios textos neotestamentarios pues algunos de ellos, además de recoger con nitidez la novedad evangélica, conservan restos de la interpretación antigua.

Encontramos diversos textos - señala Juan Pablo II-, en los cuales los escritos apostólicos expresan esta novedad, si bien en ellos se percibe aún lo "antiguo", es decir, lo que está enraizado en la tradición religiosa de Israel, en su modo de comprender y de explicar los textos sagrados, como por ejemplo el del Génesis (Juan Pablo II, 1988, n. 24, cita 49).

Así, por ejemplo, se puede recordar aquél de la Carta a los Corintios de san Pablo que afirma: "Quiero que sepáis que el varón es imagen (eikón) y gloria (dóxa) de Dios; la mujer, en cambio es gloria del varón" (1 Cor 11, 3-16); por lo que la igualdad entre el varón y la mujer no logra desprenderse de prejuicios seculares, y durante siglos muchos autores cristianos tratan de hacer compatible simultáneamente la igualdad y la subordinación. Para esta especie de "cuadratura" del círculo, se optó por afirmar que la igualdad se refiere a los bienes espirituales y la subordinación persiste en cuanto a los asuntos temporales. Como es fácil apreciar hoy, esta interpretación contradice la doble misión común que Dios encomienda al varón y a la mujer, al bendecirlos después de la creación (Gen. 1, 28: "Creced, multiplicaos, llenad la tierra y dominadla").

Analizando esta postura, la tesis de que la imagen está en el alma y esta es asexuada, presenta dificultades y es susceptible de diversas corrupciones. En primer lugar, porque la sexualidad queda restringida exclusivamente a la corporeidad (de la que con frecuencia y por diversas influencias gnósticas se ha tenido una concepción negativa), y su sentido y significado se limita a la reproducción y a la conservación de la especie, como es el caso de la sexualidad animal. Por otra parte, el varón en cuanto tal sigue conservando su condición de imagen de Dios, mientras que la mujer la tiene solo en cuanto que juntamente con el varón forma parte de la naturaleza humana; de modo que el varón solo es imagen de Dios, mientras que la mujer sola no. Ciertamente estas negaciones aparecen sobre 
todo en la argumentación teórica, en clara contradicción con la praxis de la Iglesia, en la que la mujer virgen ha tenido siempre su estatus y reconocimiento.

A la altura del siglo XX se perfila un nuevo avance en la conceptualización de la imagen de Dios: varón y mujer son, tanto juntos como por separado, imagen de Dios igualmente. En esta línea, cabe destacar los estudios sobre la imagen desarrollados por la noruega Kari E. Børresen, en los que superando viejos prejuicios, desarrolla la convicción de que tanto el varón como la mujer, en cuanto tales, son imagen de Dios. En esta línea avanzan los conocimientos antropológicos. Se ha señalado ya la transcendencia del descubrimiento científico de la fecundación y del conocimiento de la fisiología para barrer de cuajo la sombra de la supuesta pasividad femenina, que se cierne sobre la mujer desde tiempos inmemoriales.

Por otra parte, se está constatando que las diferencias no son solo biológicas o culturales, sino que también hay diversidades innatas en el plano psicológico. En este sentido el psiquiatra suizo Jung (1954/2009, pp. 49-68 y pp. 69-102), en su búsqueda de los arquetipos humanos y, en concreto, de los arquetipos femeninos del ánima y de la madre, constató que la psique humana es sexuada (no asexuada como afirmaron los medievales); observando además que los sexos no solo son complementarios entre ellos sino en el interior de cada uno, al advertir que los varones tienen dentro de su psique un "anima" que, en ocasiones, tarda en desarrollarse y que si no lo hace resultan personalidades extrañas y empobrecidas. Es decir, cada sexo tiende a desarrollar con más facilidad una vertiente de la naturaleza humana, pero tiene también una serie de actitudes, imprescindibles para humanizar la vida empezando por la propia, que solo puede desarrollar con la ayuda del otro sexo. Esta constatación es asequible a la experiencia humana ordinaria, según la cual en los varones hay potencialidades que solo son capaces de sacar a la luz las mujeres de su vida, empezando por su madre. $Y$ las mujeres tienen potencialidades (a las que Jung designó como "animus"), que solo se desarrollan si son potenciadas por los hombres de su vida, empezando por su padre. De ahí que, por ejemplo, de un varón que sepa querer se pueda decir que "ama con corazón de padre y de madre".

En tercer lugar, y debido a la acción comprometida de muchas mujeres han ido cayendo prejuicios y al haberse incorporado estas a la educación superior han evolucionado también las condiciones en los contextos laborales y sociológicos.
Por su parte, desde el punto de visto teórico, gracias a la antropología centrada en la persona, se han abierto nuevas líneas de avance, porque se ha acometido una antropología tanto filosófica como teológica partiendo del cuerpo. El cuerpo deja de ser un aspecto extrínseco a la dignidad humana, para presentarse como la expresión de la singularidad personal. Desde esta perspectiva, la condición sexuada humana, presente en la corporeidad, se comienza a ver como una expresión de la intimidad humana pasando a ser "constitutiva de la persona" y no solo "atributo de la persona", siendo su significado profundo la capacidad para reconocer a la persona y para expresar el amor. Estas propuestas están recogidas fundamentalmente en el pensamiento de Karol Wojtyla que, antes de acceder al pontificado, había desarrollado una antropología centrada en la persona, el amor y la familia que culmina en su obra sobre la Teología del cuerpo (2006). Su propuesta se sitúa más allá de los planteamientos anteriores, al afirmar que la plenitud de la imagen de Dios no se encuentra solo en la mujer y en el varón considerados independientemente, sino que está en la "unidad de los dos", lo que viene a ser una imagen de la "unidad Trinitaria". En opinión de sus biógrafos, su pensamiento en torno a esta cuestión es lo más original de sus escritos y lo que más huella dejará en el pensamiento humano. Según Weiger se trata de una bomba de relojería que se redescubrirá ya muy entrado el siglo XXI o quizá más tarde (1999, pp. 451-466). Al ser el más novedoso, nos centraremos en el análisis de su planteamiento.

\section{DOS NUEVAS CLAVES INTERPRETATIVAS}

En el horizonte intelectual de Karol Wojtyla, guiado por la experiencia vital, emergió con claridad la convicción de que el ser humano se realiza en la doble modalidad de varón y mujer. Antes, en y después de la Carta Apostólica Mulieris Dignitatem repensó esta verdad con singular penetración, sorteando prejuicios difíciles de desarraigar, reinterpretando pasajes bíblicos en los que la mentalidad "antigua" no deja aflorar con claridad la "novedad evangélica" y reabriendo puertas -ya en germen en la Escritura y en la Patrística-, que han estado cerradas en la tradición posterior.

\subsection{Inseparabilidad entre cuerpo, sexo y persona}

El punto de partida de su antropología es el cuerpo, cuestión que toma de Max Scheler, como dimensión humana visible e indiscutible. Y la clave interpretativa del resto de su desarrollo y de la originalidad de sus propuestas consiste en no separar ni el cuerpo ni 
el sexo de la persona ya que "el cuerpo es expresión de la persona" (Wojtyla, 1982, p. 238). Siguiendo la tradición oriental advierte que lo más interior se expresa en lo visible. Lo que significa también que si se cambiara el cuerpo se dejaría de ser el mismo, como también reconoce Habermas (2002, p.115). Esta es la razón en la que basa su argumentación para afirmar que el cuerpo tiene significado sacramental, al entender por tal que hace visible algo invisible.

Si el cuerpo es expresión de la persona, las características propias de la misma (unicidad y apertura relacional) deberían expresarse en la corporeidad. El ADN, el iris o las huellas dactilares expresan la irrepetibilidad y la apertura podría estar plasmada precisamente en la condición sexual. Esa podría ser la explicación de su afirmación de que "el sexo es constitutivo de persona" (Juan Pablo II, 2011, p. 78), que viene a decir que las manifestaciones sexuadas del cuerpo expresan algo más profundo que configura a la persona misma. Y cuando sostiene que el cuerpo tiene un "significado esponsal" (2011, p. 110) está expresando que la llamada al amor de la persona se refleja en el cuerpo, que materialmente es capaz de expresar el amor de dos formas distintas.

De la misma raíz nace su consideración de que el cuerpo y el sexo (la masculinidad y la feminidad frente a frente) formen parte de la imagen de Dios (Juan Pablo II, 2011, p. 97) ${ }^{1}$, porque cada cuerpo dice desde sí al otro expresando su llamada al amor y a la comunión (2011, p. 104). La condición sexuada del cuerpo es una manifestación de algo interior, que es la apertura relacional en dos direcciones complementarias de la persona, que constituye el significado profundo de la sexualidad. Por ello el sexo es constitutivo de la persona y no solo atributo suyo, y la diferencia sexuada es una realidad originaria de la que no se puede prescindir, que participa del valor absoluto y de la dignidad que tiene la persona.

\subsection{Unidad y dualidad, centro de la teología de la imagen}

Si su antropología parte del cuerpo, su teología de la imagen tiene también un novedoso punto de partida en la relectura de los textos del Génesis, donde se pone de manifiesto que la creación del ser humano fue especialmente pensada por Dios en su intimidad, en cuanto plural, es decir, es Dios Trino quien "piensa" al ser humano y a imagen de la Trinidad es creado el hombre. "Hagamos al hombre a nuestra imagen" -se afirma en el v. 26-. Parece un diálogo en la intimidad de las tres personas divinas que, como un acto libre y fecundo de su propio ser y felicidad, piensan y quieren que exista en el mundo visible una expresión de su propia vida, con su propio estilo. $Y$ tras su obra ya realizada, el Creador se complace al contemplarla cuando afirma: "Y vio Dios que era muy bueno" (v. 31).

En este sentido es significativo que en el versículo central de la creación ("Y creó Dios al hombre a su imagen, a imagen de Dios lo creó, varón y mujer los creó", Gén, 1, 27), se hable del hombre a la vez en singular y en plural. Dice primero, en singular, que Dios crea un hombre y luego utiliza el plural al referirse a la dualidad inicial de varón y mujer. En esta breve precisión, se puede encontrar ya una imagen del Creador. En efecto, la Trinidad revelada en el Nuevo Testamento tiene sus vestigios en el Antiguo, que se descubren al leer este a la luz de aquel. Así, cuando Dios Uno dice: "Hagamos" es porque en su intimidad no es un ser solitario. En Él existen tres personas distintas en el mismo nivel ontológico. La Revelación ha desvelado en el interior de la divinidad una diferencia que no altera la igualdad. En opinión de Scola (2005, p. 135), solo el reconocimiento de una diferencia de esas características posibilita reconocer la originalidad de la diversidad de la condición sexuada del ser humano.

Como se evidencia en el texto del Génesis, en "un" hombre que a la vez es "dos" parece reflejarse el mismo misterio de Dios. Tanto el "uno" como el "dos" en el hombre es originario, indeducible, la "Unidad de los dos" o "unidualidad" -de la que habla Juan Pablo II- sería una imagen de la Triunidad divina. Según esta perspectiva, la imagen divina en el ser humano acoge rasgos de la intimidad divina como la unidad y la pluralidad, la diferencia y la igualdad engarzadas entre ellas.

Sin embargo, como ya se ha señalado, la cuestión de la imagen de Dios, base inmutable de toda la antropología cristiana, no ha logrado aún ocupar el lugar central que le corresponde en la antropología teológica (Ladaria, 1993, pp. 68 ss.). Su conceptualización se ha ido abriendo paso lentamente y con dificultad en diversos contextos culturales. En este sentido es importante señalar que Juan Pablo II renueva y amplía la teología de la imagen al situarla en unas nuevas coordenadas ${ }^{2}$. La primera y fundamental consiste en descubrir, al leer el texto sagrado, que la imago Dei es una imago Trinitatis. El segundo eje es la antropología personalista que parte de la corporeidad.

La antropología personalista y la imagen trinitaria están estrechamente relacionadas y aún queda por calibrar el alcance que se deriva de su conjugación, 
que empieza por transformar la misma noción de persona y la misma teología de la imagen; lo que obliga a repensar la tradición y a ampliar los presupuestos filosóficos y teológicos necesarios para su desarrollo.

\section{DIEZ PROPUESTAS}

Los estudios de Wojtyla se apoyan en algunos presupuestos que él ya encontró iniciados y prosiguió, de los que se van derivando diversas propuestas. Señalemos algunas:

\subsection{Ampliación de la noción de persona}

El primer presupuesto son los avances en torno a la noción de persona que han tenido lugar a lo largo del siglo XX. Con ella se describe en primer lugar la unicidad de cada cual, como magistralmente expone Arendt (1958/1993, p. 200), que tiene algo de absoluto, le hace ser siempre fin y no medio, razones por las cuales debe ser amada por sí misma.

Ciertamente la noción de persona, que no conocieron los griegos clásicos, es la aportación del Cristianismo a la filosofía y nació en el intento de la inteligencia por penetrar en el misterio de la Trinidad y de la Encarnación, señalando con ella una realidad más profunda y distinta a la esencia, substancia o naturaleza que permitía profundizar en el conocimiento del ser. La diferencia en Dios se conceptualizó como persona y se describió como "relación subsistente" (Tomás de Aquino, I, qq. 29-30). La relación, un término de origen categorial, situado en otro contexto dilata su significado para expresar la diferencia personal en Dios, de un modo similar a como subsistencia no significa lo mismo que substancia. Sin embargo, el primer intento de aplicar la noción de persona al ser humano (la célebre y secularmente reiterada definición de Boecio, "Substancia individual de naturaleza racional") se apartó de esa descripción en un doble sentido, pues dejó de hablar de subsistencia para recaer de nuevo en el término substancia y no recogió el aspecto relacional. Han hecho falta muchos siglos para recuperar la dimensión transcendental de la persona, aún poco divulgada, y la perspectiva de la imago trinitatis permite integrar el aspecto relacional mediante dos avances consecutivos.

El primero ha fraguado por obra del personalismo contemporáneo que, apoyándose en la experiencia (una persona sola sería una desgracia, porque lo más propiamente humano, como el lenguaje o el amor, requieren un destinatario), ha incorporado, como constitutiva de la persona, la dimensión de apertura al otro. El progreso se recogió en la célebre frase del Concilio Vaticano II en la Gaudium et Spes, n. 24, que Juan Pablo II no se cansa de repetir: "El hombre es el único ser en el universo al que Dios ha querido por sí mismo y no encuentra su plenitud más que en el don sincero de sí a los demás". El párrafo se refiere, tanto al valor absoluto de cada persona, como al enclave relacional que desde sí misma posibilita su llamada a la comunión. La Carta Mulieris dignitatem es explícita al respecto: "ser persona a imagen y semejanza de Dios comporta también existir en relación al otro "yo" (Juan Pablo II, 1988, n. 6). Posteriormente en otros documentos del Magisterio se habla de la humanidad como realidad relacional (Congregación para la Doctrina de la fe, 2004, n. 6) y se describe a la persona como subjetividad relacional (en el Compendio de la Doctrina Social de la Iglesia, 2005, n. 149). Esta primera dilatación de la noción de persona humana, incluyendo en ella el aspecto relacional, posibilita ya profundizar en la imagen de Dios pues "es preludio de la definitiva autorrevelación de Dios Uno y Trino: unidad viviente en la comunión" (Juan Pablo II, 1988, n. 6).

\subsection{Exégesis de Génesis 2 a la luz de Génesis 1}

Otro presupuesto importante incorporado por Juan Pablo Il es la superación de la conocida y secular dificultad exegética que han presentado los dos relatos de la Creación: si se interpretaran ambos literalmente resultarían contradictorios. La hermenéutica de Juan Pablo II, que asume la diversidad de géneros literarios, propone interpretar el oscuro y arcaico texto de Génesis 2 a la luz de lo ya dicho en Génesis 1. Uno habla de la imagen, el otro -la metáfora yahvista de la costilla de Adán-, viene a ser una explicitación de la misma. En el primero se advierte que varón y mujer aparecen en la existencia juntos "desde el principio", fruto de un único acto creador (Castilla de Cortázar, 2005). La simultaneidad en el origen (ninguno es anterior al otro) viene a ser una lectura trinitaria, pues en Dios ninguna de las personas, co-eternas, es anterior o posterior, ni mayor ni menor que las otras (Denzinger-Schönmetzer, 1963, 75/39).

En consecuencia, el relato de Génesis 2 no está exponiendo cronológicamente la creación humana. Se trata de una parábola bíblica para explicar de un modo inagotable lo más profundo de la imagen trinitaria. El lenguaje simbólico, mítico o poético se revela como el más apropiado para expresar lo que es difícil de objetivar, como es el caso de la estructura de la intimidad humana. El "Adam genérico" afirma no tanto su existencia real como los dos aspectos in- 
trínsecos de la persona: el autoconocimiento al reconocerse superior al mundo natural (primer significado de la soledad originaria) y la llamada a vivir para otro, en comunión; segunda dimensión de la persona, que solo puede formarse sobre la base de dos soledades. La parábola explica también la razón por la cual fueron dos "desde el principio", porque "no es bueno que el hombre esté solo" $(2,18)$. En este sentido afirma la Gaudium et Spes, n. 12: "Pero Dios no creó al hombre dejándolo solo; desde el principio varón y mujer los creó (Gen 1, 27), y su unión constituye la primera forma de comunión de personas". A lo que Juan Pablo II añade: “

El ser humano no puede existir "solo"; sólo puede existir como "unidad de los dos" y, por consiguiente, en relación con otra persona... Ser imagen y semejanza de Dios conlleva, por tanto, también existir en relación con el otro "yo" $(1988$, n. 6).

Una lectura conjunta de ambos pasajes supone el enorme acierto de introducir un sesgo sorprendentemente nuevo, que supera una milenaria interpretación que ha dado lugar a reiterados errores y devenido obstáculos hasta hoy difícilmente salvables, a la hora de calibrar la diferencia varón-mujer. La exégesis de Juan Pablo II, asumiendo los logros de la hermenéutica moderna, supone un cambio de rumbo que introduce en el discurso de la antropología teológica un nuevo y luminoso punto de partida.

\subsection{Superación del mito del andrógino}

Desde esta nueva interpretación del Génesis se clarifican debates que han durado siglos. Entre ellos es importante el mito del andrógino. La exégesis literal que la tradición de Israel hizo de Génesis 2 se unió a la concepción del inicio del género humano recogido por Platón en El Banquete, 189c-193d, y el Adán solitario se interpretó a la luz del andrógino del mito de Aristófanes. Este, originariamente uno, fue dividido por un castigo de la divinidad y cada parte sufriente busca su otra mitad para recuperar su identidad. Aunque en la Revelación bíblica se pone de manifiesto que la diferencia sexual no tiene que ver ni con el pecado ni con el castigo, sí se ha concebido que, tras el sueño del Edén, el primer "Adam" se convirtió en un varón y una mujer (ambos incompletos), cada uno mitad de la humanidad. El planteamiento complica la concepción ya errónea de la complementariedad que se derivaba de la lectura literal de Génesis 2, en el que solo la mujer era complemento, hasta tal punto que esta categoría sigue aún hoy en tela de juicio ${ }^{3}$.
Juan Pablo II, desde su visión personalista, hace un planteamiento diferente. La mujer aparece en la Creación como "el otro yo en la humanidad común" (1988, n. 6). Otro yo es un modo de decir "otra persona". Y una persona es ya un "todo" con valor por sí mismo, dueño de sí, libre y responsable con independencia de los demás. Lo que aparece como común es la humanidad. Las dos personas, sin embargo, tienen una característica: están orientadas una a la otra, están llamadas a la unidad, a lo que responde la expresión "Unidad de los dos", que no elimina la unicidad personal de cada uno sino que la presupone. Por tanto, en torno a una correcta antropología habría que distinguir dos niveles de unidad: la personal (momento de la soledad, frente a Dios y frente al cosmos) y la "unidad de los dos" que trascendiendo las personas se sitúa en otro orden de unidad que acoge la diferencia y la pluralidad.

Pues bien, la interpretación del origen humano que se deriva de la "unidad de los dos" posibilita la superación lisa y llana (definitiva) de la influencia del andrógino. No solo es un planteamiento diferente al del andrógino, sino justamente el contrario: no es uno que se divide en dos, sino dos que se hacen uno. Enfocando al revés el punto de partida, el horizonte abierto se despliega en un crescendo hasta alcanzar otra dimensión ontológica. La unidad de los dos permite acceder a otro orden transcendental, insospechado e inalcanzable desde el planteamiento griego.

\subsection{La imagen trinitaria y la misión común}

Para desentrañar las implicaciones de la imagen trinitaria es imprescindible también recoger el contenido de Génesis 1, 28: "Y los bendijo Dios y les dijo: "Creced, multiplicaos y llenad la tierra y sometedla". Se trata de la misión a realizar en común, que a su vez es doble: la familia, a través de cuya fecundidad se Ilenará la tierra, y el trabajo para cuidar y dominar el mundo y construir la cultura y la historia.

La tarea encomendada adquiere un significado peculiar, porque una imagen trinitaria parece indicar que debería consistir en una unidad de tres. La pregunta surge inmediata ¿por qué unidad de dos y no tres? La respuesta se va esclareciendo al considerar que la imagen no consiste en ser el modelo sino en reflejarlo. Que la criatura no es Dios se expresa en que no es TRES desde el principio. Sin embargo, la imago trinitatis se manifiesta en que la bendición abre el dos al TRES, a través del dinamismo de la acción, que se funda en la donación desinteresada de cada uno en el amor. La dualidad no se clausura en sí misma sino que trascendiéndose de esa "unidad de los dos" brota una peculiar fecundidad. 
Misión común significa, por otra parte, que el tres no es resultado de la acción de uno o de otra por separado, sino de la unidad de los dos. En este aspecto sí se puede decir que cada uno aporta la mitad. Lo que resulta evidente en la transmisión de la vida es también necesario en el cuidado y transformación de la tierra. De un modo similar a como, sin la aportación diferencial de los dos en la familia no habría fecundidad, así sin los recursos de la feminidad o de la masculinidad no sería posible un desarrollo sostenible en el ámbito social, económico y cultural. Juan Pablo II lo constata:

El texto de Génesis 2, 18-25 indica que el matrimonio es la dimensión primera y en cierto sentido, fundamental de esta llamada (a existir recíprocamente). Pero no es única. Toda la historia del hombre sobre la tierra (...) se desarrolla en la integración en la humanidad misma, de lo "masculino" y de lo "femenino" $(1988$, n.7).

En la procreación la estructura donal del varón y de la mujer se transforma en paternidad y maternidad y en la familia aparece la tríada: padre-madre e hijo, que evidencia una imagen trinitaria. La unidad en la acción "no refleja una igualdad estática y uniforme, ni una diferencia abismal e inexorablemente conflictiva" sino una relación vivenciada como "un don enriquecedor y responsabilizante" (Juan Pablo II, 1995, n. 8). Y lo específico de cada uno no consiste en la diversidad de funciones sino más bien en el modo de realizar una misma función, en los matices que la condición de cada uno encuentra para la solución de los problemas con los que se enfrenta, e incluso en el descubrimiento $y$ en el planteamiento mismo de esos problemas (Escrivá de Balaguer, 1968/1998, p. 184).

En definitiva, el TRES, que no está dado desde el principio, se presenta como tarea. Sin embargo, como la acción en último término hunde sus raíces en la ontología personal, por ello, el tres humano sigue llevando el sello de la dualidad, lo que se manifiesta en la familia en que el hijo volverá a ser también o varón o mujer. La diferencia triádica radical solo se da en la intimidad de Dios.

\subsection{La "comunión de las personas", plenitud de la imagen}

Una vez afirmada la "unidad de los dos" que se trasciende en el tres, seguimos preguntando por el contenido de la imagen. En la evolución doctrinal de la imago Dei se puede constatar en una primera etapa que para los hebreos solo Adán era imagen, Eva era derivada. En la tradición cristiana para acoger a la mujer dentro de la imagen, cuajó la interpretación (en sí misma un avance), de que la imagen estaba en el alma y esta era asexuada. A la vez, el varón seguía siendo imagen, mientras que la mujer lo era únicamente en tanto que unida al varón, pero ella sola no. Finalmente, la mujer en cuanto tal es admitida también dentro de la imagen. Cabría destacar que es en la Mulieris dignitatem, cuando por primera vez en el Magisterio se afirma explícitamente que la mujer en cuanto mujer es imagen de Dios ${ }^{4}$. Se trata de una afirmación reiterada, como para reafirmar una verdad anteriormente ausente.

En el progresivo desarrollo de la imagen, Juan Pablo Il va desde la Persona a la Comunión.

El hombre se convierte en imagen de Dios -afirma-, no tanto en el momento de la soledad cuanto en el momento de la comunión. Efectivamente, él es "desde el principio" no sólo imagen en la que se refleja la soledad de una Persona que rige el mundo, sino también, y esencialmente, imagen de una inescrutable comunión divina de Personas (2011, pp. 73-74).

Ciertamente cada persona es imagen en cuanto ser racional y libre, capaz de conocer y amar a Dios. Desde aquí se ha desarrollado la teología de la imagen en la tradición occidental. De la mano de San Agustín, cuya influencia ha sido decisiva durante siglos, por medio de la llamada "analogía psicológica", la imagen trinitaria se refleja en cada persona a través de las diferencias de sus potencias: memoria, entendimiento y voluntad. Esta ingeniosa analogía tiene el inconveniente de que, si excluye otras, dejaría fuera de la imagen las relaciones interpersonales lo que supone un recorte importante de la realidad humana como señala Ratzinger (1976, p. 173). De hecho, Agustín de Hipona consideró una opinión errónea la analogía familiar tal y como se formulaba en su tiempo, y tras él la puerta quedó cerrada 5 .

A la vuelta de los siglos, Juan Pablo II vuelve a constatar que la persona aislada no solo no agota la imagen trinitaria sino que no constituye su plenitud. La "unidad de los dos", signo de la comunión interpersonal, es parte y parte importante de la imago Dei. Esta idea supone un significativo avance, para Scola una tesis clave, no explorada completamente por la Teología, "en la que se puede entrever una de las aportaciones más significativas del Magisterio papal, cuyo alcance abarca todo el campo de la teología dogmática" (2005, pp. 134-135), que está pidiendo una peculiar ontología para la antro- 
pología. Desentrañar la riqueza antropológica que encierra requiere diversos ángulos. A continuación subrayaremos que la comunión fruto del amor se produce en reciprocidad.

\subsection{La reciprocidad, requisito de la igualdad}

Si ser persona es vivir en relación a otro, el vivir "para" de cada uno engendra reciprocidad que es, ante todo, una afirmación de la persona y supone, también, un reconocimiento de la pertenencia de ambos al mismo nivel ontológico. Que el "Adam" del Paraíso no encontrara compañía hasta que apareció ante sus ojos alguien que era "carne de su carne y hueso de sus huesos" (Gén 2, 18) marca un nivel de igualdad, que nunca se puede perder de vista. En este sentido la Mulieris Dignitatem no deja lugar a la ambigüedad:

Sólo la igualdad -resultante de la dignidad- de ambos como personas, puede dar a la relación recíproca el carácter de una auténtica "comunio personarum" (Juan Pablo II, 1988, n. 10).

En torno a esta cuestión lo más destacable es que algunos textos bíblicos parecieran expresar la sumisión unilateral de la mujer por lo que, una vez más, adquiere singular importancia la clarificación de Juan Pablo II al respecto, donde constata que "encontramos diversos textos en los cuales los escritos apostólicos expresan esta novedad, si bien en ellos se percibe aún lo "antiguo", es decir, lo que está enraizado en la tradición religiosa de Israel, en su modo de comprender y explicar los textos sagrados, como, por ejemplo, el del Génesis capítulo 2". Y concluye al hilo del análisis, la analogía del amor esponsal Cristo-Iglesia de la carta a los Efesios, que "en relación a lo "antiguo", esto es evidentemente "nuevo": es la novedad evangélica. (...) El desafío del "ethos" de la redención es claro y definitivo. Todas las razones en favor de la "sumisión" de la mujer al varón en el matrimonio se deben interpretar en el sentido de una sumisión recíproca de ambos" (1988, n. 24).

Llegados a este punto, es necesario repetir con Juan Pablo II que todas las interpretaciones que rompan la reciprocidad no pertenecen a la novedad evangélica ni al mensaje bíblico, sino al lastre del envoltorio cultural que lo condiciona. Como acentúa el papa, esa novedad evangélica ha de irse abriendo camino para cambiar las mentes y los corazones, erradicando una mentalidad que resulta más costosa y lenta de superar que la ardua carrera que supuso abolir la esclavitud.

\subsection{La complementariedad ontológica, clave de la diferencia}

Tras asentar la reciprocidad en las relaciones sexuadas, paso primero y fundamental, es preciso seguir avanzando. Para ello voy a partir de la afirmación de otro documento eclesial, la declaración Inter insigniores (15-X-1976) en el que se sostiene que la diversidad sexuada "determina la identidad propia de la persona y esa distinción se ordena no solo a la generación sino a la comunión de personas" (Congregación para la Doctrina de la fe, 1976/1978, p. 53). En ella se entrevé que la communio personarum intratrinitaria y la que se da entre varón y mujer se asemejan en ser una comunión entre personas relacionalmente distintas.

La categoría para expresar la diferencia utilizada por Juan Pablo II no es otra que la de complementariedad. Con sus nuevas claves comienza hablando de complementariedad recíproca, corrigiendo así la interpretación según la cual solo la mujer era complemento y esa noción, despejados los inconvenientes precedentes, amplía su significado adquiriendo progresiva relevancia, hasta afirmar que "la complementariedad no solo es biológica y psicológica, sino también ontológica" (1995, n. 7). Refiriéndose a la complementariedad ontológica explica que "cuando el Génesis habla de ayuda no se refiere solamente al ámbito del obrar, sino también al del ser" (1995, n. 7). Nos encontramos en el nivel constitutivo, que viene a dar razón del orden operativo. Juan Pablo II explicita, además, que el significado de la "unidad de los dos" consiste en una "unidualidad relacional" (1995 n. 8). La relacionalidad, por tanto, aparece como la llave para explicar la unidad de iguales en la diferencia. Ahora bien, la cuestión estriba en saber cómo descubrir la diferencia en las relaciones. Volviendo al pasaje de Génesis 2 , una vez asentada la simultaneidad en el origen, en Adán se descubre cierta razón de principio (origen) y en Eva cierta razón de fin y se podría concluir que el significado más profundo de la parábola yahvista (Eva procediendo del costado de Adán), consistiría en que está revelando una relación de procedencia en el origen, análoga a las existentes en el seno de la Trinidad, que los configura como personas distintas.

En el ámbito antropológico las relaciones se descubren en el obrar que permite llegar al ser. De esta manera, el modo de procrear presenta de una manera plástica la maternidad como relación diferente de la paternidad. El varón al darse sale de sí mismo y saliendo de él se entrega a la mujer y su don se queda en ella. La mujer se da pero sin salir de ella, acogiendo en ella. Su distintos modos de darse uno al otro son 
complementarios, pues sin la mujer el varón no tendría donde ir y, a su vez, sin él ella no tendría a quien acoger. La diferencia entre estas dos relaciones consiste en estar orientadas una a la otra, lo que posibilita a la vez la "unidad de los dos", pues si ambas estuvieran abiertas en la misma dirección correrían paralelas pero sin encontrarse.

Expresar ontológicamente esas diferencias está reclamando una segunda ampliación en la noción de persona para acoger la complementariedad que da lugar a la unidad dentro de la apertura relacional. Se trata de la estructura esponsal de la persona. Teniendo en cuenta que las relaciones no tienen nombre de naturaleza, los términos gramaticales apropiados para expresarlas no son los sustantivos, ni tampoco los pronombres que se refieren a las personas, sino las preposiciones. Entre ellas, al varón le correspondería la preposición desde, pues parte de sí para darse a los demás y a la mujer la preposición en, pues se abre dando acogida en sí misma. Integrada en la apertura relacional general, la persona varón se podría describir, entonces, como "ser-con-desde" o "ser-para-desde", y a la mujer como "ser-con-en" o "ser-para-en". De este modo, el acto de ser humano, descrito de un modo general como "ser-con" o "ser-para", acogería la díada. Observada desde la imago trinitatis, la estructura esponsal es una manifestación de riqueza ontológica, pues constituye la base que explica por qué la plenitud humana solo se encuentra en el don de sí.

\subsection{La analogía familiar}

Ya se ha señalado que padre-madre-hijo, en cuanto relación triádica, se convierte en una imago Trinitatis.

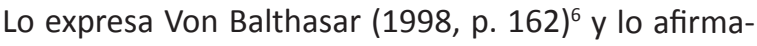
ron los Padres de la Iglesia anteriores a San Agustín 7 . Juan Pablo II redescubre la analogía familiar por otro camino. Pensando en la intimidad de Dios constata sus lazos familiares. Ya en 1979 afirmaba:

Dios en su misterio más íntimo no es una soledad sino una familia, puesto que lleva en Sí mismo paternidad, filiación y la esencia de la familia que es el amor. Este Amor, en la familia divina, es el Espíritu Santo" (Homilía, 28-I-1979).

Y más adelante apoyándose en Ef 3, 14-15: “Del Padre toma nombre toda familia en el cielo y en la tierra" expone que "a la luz del Nuevo Testamento es posible descubrir que el modelo originario de la familia hay que buscarlo en Dios mismo, en el misterio trinitario de vida» $(1994$, n. 6).
Volviendo a la consideración de que el tres, al que está abierta, tiene que estar de algún modo dentro de la díada como potencialidad, se plantea la pregunta acerca del descenso ontológico del tres al dos. En otras palabras, si padre-madre-hijo, en cuanto relación triádica es una imago Trinitatis ¿de qué modo paternidad, maternidad y filiación se contraen en la dualidad varón-mujer? La respuesta podría estar en que la filiación, respecto a sus progenitores y a Dios, es una relación constitutiva tanto en el varón como en la mujer. En cuanto hijos no se diferencian sino más bien son idénticos, por lo que se podría sostener que su diferencia radicaría en la presencia en cada uno de la paternidad o de la maternidad (entendidas estas como potencialidades y modo de contribuir al bien de los demás). Ser persona varón coimplicaría a la vez filiación y paternidad y ser mujer filiación y maternidad. En conclusión, la apertura de la persona humana no sería simple como la de cada persona divina, sino la suma de dos relaciones constitutivas. Sea como fuere, se puede decir que también la estructura esponsal (varón-mujer) tiene, en definitiva, un carácter familiar.

\subsection{El significado esponsal del cuerpo}

Juan Pablo II, descubre que el cuerpo, a la par de ser expresión de la persona y manifestación visible de la intimidad personal, tiene un significado esponsal. $Y$ por esponsal entiende que el cuerpo es precisamente lo que permite reconocer y afirmar al otro como persona, teniendo a la vez la capacidad para expresar el amor. En palabras de Wojtyla:

El cuerpo humano, orientado interiormente por el "don sincero" de la persona, revela no sólo su masculinidad o feminidad en el plano físico, sino que revela también este valor y esta belleza de sobrepasar la dimensión simplemente física de la "sexualidad". De este modo se completa, en cierto sentido, la conciencia del significado esponsal del cuerpo, vinculado a la masculinidad-feminidad del hombre. Por un lado, este significado indica una capacidad particular de expresar el amor, en el que el hombre se convierte en don; por otro, le corresponde la capacidad y la profunda disponibilidad para la "afirmación de la persona" (2011, p. 110).

Por ello, los modos de expresar el amor se despliegan en un amplio abanico, no solo la una caro; pues los gestos, la mirada, la sonrisa, las caricias pueden expresar y expresan el amor, a un esposo, a un hijo, a un amigo o a otra persona. La masculinidad y la feminidad presentes en la corporeidad permiten descubrir aspectos importantes de la imagen de Dios. 
En su reciprocidad esponsal y fecunda, (...) su relación más natural, de acuerdo con el designio de Dios, es la "unidad de los dos", o sea una "unidualidad" relacional, que viene a ser una imagen de la Unidad de los Tres (Juan Pablo II, 1995, n. 8).

En este nuevo modo de plantear la cuestión, el cuerpo recupera su inclusión dentro de la imagen de Dios. Y no solo el cuerpo masculino, cuestión que nunca ha sido puesto en duda por cuanto que en la Encarnación el Hijo de Dios asume una naturaleza de varón, sino que también la mujer en cuanto tal es considerada como imagen de Dios, incluyendo su corporeidad.

\subsection{Transversalidad de la condición sexuada}

Centrémonos ahora en otras palabras de Karol Wojtyla: "la sexualidad es riqueza de toda la persona -cuerpo, sentimiento y espíritu-, que manifiesta su significado íntimo al llevar a la persona hacia el don de sí misma en el amor" (1981, n. 37; 1995, n. 97), para analizar una característica muy peculiar de la condición sexuada, su carácter en cierto modo omniabarcante. Abarca todo el ser humano: cuerpo, sentimientos y espíritu y todos los aspectos de cada una de esas dimensiones. Así se puede comprobar respecto al cuerpo, en el que la diferencia sexual $X X$ o $\mathrm{XY}$, calculada por los genetistas en un $3 \%$, tiene la característica de que se halla presente en cada célula del cuerpo; de modo que todo el cuerpo es sexuado, no solo unos órganos con una función concreta. Pero también son sexuadas las percepciones, emociones y pensamientos: esa diferencia tiñe toda la naturaleza humana, cuerpo y mente. Esta transversalidad ha presentado a los filósofos, y sigue haciéndolo, muchas dificultades a la hora de establecer su estatuto ontológico, pues no es un accidente ni da lugar a una esencia distinta. Esa transversalidad, presente también en la acción, se resiste por tanto a ser clasificada tanto como forma accidental o substancial. ¿Cómo catalogarla, entonces, si no es forma ni "esencia"? Hay diversas tesis al respecto ${ }^{8}$.

Ya se ha señalado la tesis de Jung de que los sexos no solo son complementarios entre ellos, sino que lo son también dentro de ellos, al advertir que los varones tienen en su psique una dimensión femenina: "anima" y correlativamente las mujeres una masculina: "animus". El profesor Polo la explicaba como una tipicidad o reparto de las potencialidades de la esencia humana (2011). Al considerar que la esencia humana tiene tanta riqueza de posibilidades que ningún individuo concreto podría tenerlas todas, algunas diferencias y semejanzas entre personas se clasifican por temperamentos, profesiones o caracteres, que agrupan a seres humanos con las mismas o muy parecidas características. Se trata de los tipos humanos que vendrían a ser como un reparto de las perfecciones de la naturaleza; de un modo similar a cómo Gardner (2011) habla de inteligencias múltiples, distinguiendo nueve tipos, y no todas las personas tienen los nueve. Pues bien, entre las diversas tipicidades, Polo señala que la principal, en cuanto reparto de la esencia, es la que se da entre varón y mujer. A primera vista la propuesta resulta convincente, pues solamente si se tienen en cuenta masculinidad y feminidad conjuntamente se alcanza la humanidad completa. Pero si se repasa, por ejemplo, la caracterología, ser apasionado, colérico, flemático o melancólico no depende de la condición sexuada. Tampoco depende de ella tener inteligencia musical, emocional, creativa o naturalista, pues aparte de que los talentos son personales, en todos los tipos humanos hay varones o mujeres. De ahí que la transversalidad que observamos en la diferencia sexuada no parece que sea solo tipicidad.

Otra tesis apunta a un reparto entre cualidades o virtudes femeninas o masculinas. No es infrecuente encontrar quien afirma que corresponde a las mujeres la ternura y a los varones la fortaleza. Pero si el asunto se contempla de cerca, los hábitos y las virtudes pertenecen a la naturaleza y tanto el varón como la mujer tienen una naturaleza humana en cierto modo completa, a cuya perfección pertenece el cultivo de todas las virtudes que, como vasos comunicantes, se correlacionan entre ellas; por lo que tampoco resulta convincente esta explicación.

Por último, existe otra propuesta que, a partir de los datos genéticos, desarrolla la tesis de la modalización sexual según la cual varón y mujer son dos modos diversos de realizar la misma naturaleza. Así, las virtudes o los talentos cristalizarían con matices diferentes en varones y en mujeres. Tener buena voz, por ejemplo, además de ser un talento personal, se manifiesta en cuatro tonalidades, de las cuales, aunque haya dos comunes, solo los varones alcanzan la más baja y las mujeres, la más alta.

La diferencia se presenta entonces, como un modo distinto de hacer lo mismo, dos modos distintos de solucionar problemas o incluso de ver y descubrir los problemas (hay cosas que ven los hombres y no tanto las mujeres, pero la inversa es completamente verdadera); por lo que el concurso de ambas perspectivas se torna indispensable cuando un asunto quiere resolverse atendiendo a todas sus dimensiones. 
Ante una diferencia tan escurridiza, filosóficamente hablando ¿cómo catalogar esa transversalidad que se hace presente en el resto de las diferencias humanas? ¿Es posible que el dimorfismo sexual humano no sea una forma sino que se trate de una diferencia de otro orden distinto que trasciende las esencias? Esto podría ser plausible si se tienen en cuenta los actuales avances en la conceptualización de la noción de persona, pero en concreto para conceptualizar esta transversalidad nos encontramos con una gran dificultad: la cosmovisión monolítica de la realidad, a la que ahora me referiré.

\section{DOS TAREAS PENDIENTES}

Si hasta aquí se han señalado los avances y obstáculos ya removidos, llega el momento de señalar los puntos oscuros, los escollos pendientes de remover; o mejor, las tareas pendientes, para que se puedan llevar hasta sus últimas consecuencias las coordenadas trazadas.

\subsection{Cosmovisión monolítica de la realidad}

La filosofía del UNO monolítico, versión ontológica de la estructura del Cosmos, es el principal escollo para pensar la diferencia de la condición sexuada entre varón y mujer. En filosofía nunca se ha reconocido una diferencia trenzada con la igualdad. Es ilustrativo advertir que cuando los hombres han pensado a las mujeres, o bien las han sublimado poniéndolas más arriba, o las han subordinado poniéndolas más abajo. Otras veces las han puesto detrás o quizá delante, pero nunca AL LADO. Y cuando las mujeres han querido afirmar su identidad, las alternativas ensayadas han sido: imitar al varón, o bien competir con él y suplantarlo; o bien trivializar o anular la diferencia. La causa de no encontrar una propuesta adecuada es la ausencia de una filosofía que haga sitio al DOS diferente en el mismo nivel. En ese caso solo resta minusvalorar, imitar, rivalizar o negar a uno para poder afirmar al otro. Esta dificultad de fondo, que subyace en nuestra cultura ha sido puesta de relieve por mujeres filósofas y teólogas a la vez, como es el caso de Elisabeth Schüssler Fiorenza, que trabaja en Harvard.

Lo cierto es que para proseguir profundizando en la novedad aportada por Juan Pablo II en el Magisterio, de que la "unidad de los dos" es una imagen de la "unidad de los tres" es necesario profundizar en la Unidad transcendental. Esta dificultad ha sido advertida por Leonardo Polo, quien en mi opinión ha puesto las bases para resolverla, mediante su propuesta de ampliación transcendental: la antropología tiene un ámbito transcendental propio donde el ser personal es descrito como co-existencia. Afirma que dicha ampliación afecta sobre todo al transcendental UNIDAD, no resuelto aún por ninguna filosofía (2008, pp. 20-29). En su opinión, la Unidad no puede ser ni Monolítica (soledad, individualismo), ni el Todo (panteísmo o colectivismo), sino que en su seno ha de acoger la DIFERENCIA, que haga posible explicar el amor interpersonal y la unión (CO-SER) que posibilita. Eso supone ampliar toda la cosmovisión en la que se han fundado hasta ahora las civilizaciones occidental y oriental. En este sentido considero interesante profundizar en propuestas como la antropología transcendental del propio Polo (1999/2003), quien propone y desarrolla una ontología propia y peculiar de la persona, diferente de la del Cosmos, ampliando la metafísica clásica y articulando simultáneamente la distinctio realis y la diferencia heideggeriana. Afirma que el Cosmos en su conjunto tiene un solo acto de ser y que la persona es irrepetible porque cada una tiene el suyo propio. La persona se distingue del Cosmos tanto en su acto de ser, que es libre, como en su esencia, que es capaz de hábitos. De esta forma, de un modo similar a como la filosofía clásica distinguió una serie de propiedades transcendentales del ser, el acto de ser personal tendría sus transcendentales propios: la libertad, la inteligencia, el amor. Adquiere singular relevancia su desarrollo de la libertad transcendental y parece necesario que elaboraciones teóricas como esta que solidifican los descubrimientos personalistas, aún poco divulgadas, se vayan abriendo camino.

Sin embargo, Polo, aunque enfatiza la apertura personal y la importancia de la dualidad no desarrolla la dualidad dentro de la constitución personal, ni termina de desplegar la diferencia en el orden transcendental.

En este sentido la ontología peculiar de la "unidad de los dos" requiere el desarrollo de una filosofía de la DÍADA. Distinguido el cambio de nivel ontológico y tras la inclusión de la apertura relacional en la descripción personal, la articulación de una diferencia que no lesione la igualdad, está pidiendo una segunda ampliación de la noción de persona que dé cuenta de la estructura esponsal. Asentado el nivel de la unidad personal queda pendiente articular una ontología que explique un orden de unidad que incluya la diferencia en el mismo nivel transcendental, una diferencia de carácter relacional. Con una filosofía de la DÍADA se podría llegar al último estrato de la estructura personal para poder focalizar la identidad masculina como diferente a la identidad femenina, 
como dos personas distintas. Este podría ser el nivel ontológico, peculiar de una antropología que no se hiciera al margen de la diferencia sexuada que representa a la humanidad completa.

En tercer lugar, una ampliación de esta cuestión necesaria para explicar la familia sería un estudio, aún no hecho, sobre las relaciones triádicas. Si bien un varón y una mujer tienen capacidad para formar una "unidad de dos", un "co-ser" por el que llegan a ser algo que no pueden ser cada uno por separado; sin embargo, dicha unidad da lugar a una fecundidad, no solo biológica sino cultural y de desarrollo humano, que está abierto al tres. Así en la familia, las personas se convierten en "padre" y "madre" gracias a un "hijo". De este modo; la familia está intrínsecamente constituida por una relación triádica según la cual solo se puede ser padre si hay hijo y madre, solo se es hijo si hay padre y madre, solo hay madre si hay padre e hijo. Se trata, pues, de una relación de tres términos en los que cada uno es determinado por dos extremos distintos a él.

\subsection{Los límites de la analogía esponsal}

Pasemos finalmente a detectar y comentar una segunda tarea pendiente de gran calado antropológico y teológico. Se ha comentado al inicio que, en la cuestión de la imagen de Dios, Wojtyla da un paso más respecto a los adelantos que ya se habían producido en su siglo. Sin embargo, Kari E. Børresen ha escrito que la Mulieris dignitatem presenta una imago Dei actualizada y una tipología anticuada (1992, pp. 181195). ¿A qué puede deberse este juicio, teniendo en cuenta que avanza más que la autora? La teóloga noruega se está refiriendo a una de las dificultades que presenta la hermenéutica de la simbología bíblica para articular la diferencia antropológica. En ella se advierte que el Esposo y la esposa representan niveles ontológicos jerarquizados, pues el Esposo siempre es Dios o Cristo, y la esposa la humanidad; y de su lectura literal se deriva que ser esposa es sinónimo de ser criatura, cuestión que es preciso superar. Si esta consecuencia, que aparece como inevitable, se reviste de la cosmovisión del prestigio del uno con la subordinación subsiguiente, parece justificar que se haya hablado del principio (incluso del derecho) de la capitalidad del varón como signo de preeminencia. La misma jerarquización se advierte en la elaboración teológica de María como la nueva Eva, ante Cristo nuevo Adán. La primera limitación de la dimensión simbólica es, por tanto, que desdibuja la reciprocidad en igualdad.
Pero esa no es la única ni la principal limitación de esa analogía. Hay otra de más calado y más difícil de superar: que en la esposa se incluyen tanto los varones como las mujeres, impidiendo distinguir una diferencia que se busca como radical. La tesis mística, especialmente presente en san Juan de la Cruz, según la que el varón se sitúa ante Dios como esposa tiene un sentido espiritual, que resulta forzado trasladar a la antropología, pues Cristo, esposo, es ante todo el modelo de los varones, también en el modo de tratar a las mujeres. Sin embargo, en su discurso, Juan Pablo II, tal vez por el influjo de esa mística, no deslinda ambas afirmaciones, que antropológicamente resultan incompatibles al representar cada una un término opuesto de la misma relación. No obstante, en su pensamiento sí encontramos la solución para poder superar estas limitaciones, que no es otra que seguir utilizando la misma estrategia hermenéutica, usada al rehacer la exégesis de los pasajes del Génesis, para reinterpretar la analogía esponsal. Como se ha señalado, Juan Pablo II emplea el nexus misteriorum, contemplando los demás a la luz del de la Trinidad.

La propuesta está incoada en Von Balthasar que, convencido de que la distinción varón-mujer participa de la imagen de Dios señala que: "Si no fuera así, Cristo no podría servirse de la relación de los sexos para representar el misterio de la Iglesia" (1994, p. 76).

Partiendo de la imagen de Dios como imago Trinitatis, si ser mujer en cuanto tal es imagen de Dios, ser esposa (otro modo de decir mujer) no debería estar fuera de la imagen divina. Por otra parte, no faltan textos en la Sagrada Escritura en los que Dios, en la personificación de la Sabiduría, también se presenta como esposa y madre (Sb 8, 2; 8, 9; Sir 14, 26-27; 15,16; 24, 18-21; por todo lo cual sería legítimo preguntar por el arquetipo divino a imagen de quien está creada la mujer. Y en cuanto al varón, se podría poner en relación la enseñanza paulina de que Dios Padre es el principio de toda familia en los cielos y en la tierra (cfr. Ef 3, 14-16), con la razón de principio que en el texto yahvista se descubre en el varón, recolocando así todas las interpretaciones jerárquicas en torno a su capitalidad; porque el que en Dios Padre tome principio la divinidad no implica que sea antes o que tenga mayor dignidad que las otras personas de la Trinidad.

La Mulieris dignitatem, n. 29 insinúa una argumentación en la línea trinitaria al hablar del "orden del amor", en cierto modo paralelo al orden (taxis) de las personas en Dios, que no es temporal. El orden del amor -afirma- pertenece... a la vida trinitaria. En la vida íntima de Dios el Espíritu Santo es la hipós- 
tasis personal del amor. Mediante el Espíritu, Don increado, el amor se convierte en un don para las personas creadas». Juan Pablo II continúa haciendo un paralelismo entre el Espíritu Santo y la mujer: «La llamada a la existencia de la mujer al lado del varón (...) ofrece en el mundo visible condiciones particulares para que el amor de Dios se derrame en los seres creados a su imagen». $Y$, aunque no dice cuáles son esas condiciones particulares, el Pontífice concluye distinguiendo entre el modo de amar del esposo y la esposa según un orden: el esposo es el que ama para ser amado; y la esposa, la que recibe amor para amar a su vez $(1988$, n. 29).

Desde estas premisas cabría preguntarse si el misterio de Dios es un misterio de amor entre personas distintas, quizá no todas ellas amen de la misma manera; porque además, el orden del amor esponsal tampoco tiene carácter temporal sino más bien relativo. Si el don del esposo es amar primero, el don de la esposa será su correlato. De todo lo cual se puede concluir que si ser esposa es imagen de Dios, el don de la esposa consistirá también y, sobre todo, en amar como ama Dios.

Por su parte, desarrollar la eclesiología desde la imago Trinitatis podría dar lugar a resultados sorprendentes, que acogerían datos dispersos en la Patrística. Para San Metodio del costado abierto de Cristo salía no tanto la Iglesia sino el Espíritu que, fruto de la Cruz, formaría la Iglesia ${ }^{9}$. La analogía esponsal interpretada desde la reciprocidad completaría a Cristo-cabeza con el Espíritu Santo-alma de la Iglesia y a nivel humano habría que profundizar en las dimensiones mariana y apostólica-petrina para encontrar finalmente el paralelismo entre ambos niveles; pues no en vano, según el Apocalipsis, la esposa y el Espíritu claman en la misma dirección (Ap. 22, 20).

Actualmente es esperanzador el nuevo giro que aporta Ángelo Scola a la hermenéutica esponsal en vistas a explicar la familia, advirtiendo que el "misterio nupcial" encierra una experiencia antropológica originaria que todos conocemos, de la que todos sabemos aunque, como todo lo que tiene que ver con el fundamento, se resiste a ser objetivada. Dicha experiencia se estructura en tres momentos: diferencia sexual, donación sincera de cada uno y fecundidad, donde el primer elemento adquiere su pleno sentido junto a los otros dos.

En definitiva, la propuesta consiste en repensar la analogía esponsal desde la imagen de Dios como imago Trinitatis, que incluiría repensar la diferencia en Dios, a lo que ayudaría la analogía familiar, incorporando a la hermenéutica de Génesis 2 las intuiciones de la patrística anterior a San Agustín y esclareciendo el arquetipo de la maternidad en Dios.

\section{NOTAS}

1. Recupera así una tradición del siglo II. San Ireneo afirma que: "el hombre es imagen de Dios incluso en su corporalidad" (Adversus Haereses, V, 6, 1; $\mathrm{V}$, 9, 1-2)

2. El Concilio Vaticano II, con la Constitución Gaudium et Spes $(12,22)$ supuso un viraje señalando el camino de la recuperación de la teología de la imago Dei, relacionando Antropología y Cristología, que enlaza con los primeros desarrollos patrísticos. Juan Pablo Il da un paso más relacionando la Antropología con la Trinidad a través del misterio de la Creación, deslindando esta del pecado posterior
3. Scola omite este término para separarse del andrógino y habla solo de "Reciprocidad asimétrica", entendiendo por asimetría el abanico de relaciones diferentes, que surgen de la "unidad de los dos", casi todas derivadas de los lazos familiares: esponsalidad, paternidad, maternidad, filiación, fraternidad, amistad... (2001, pp. 136-138, entre otros lugares).

4. Cfr. nn 6 (tres veces), 7 y 10, entre otros.

5. Cfr. San Agustín (De Trinitate, XII, $5,5 ; 7,9 ; 8,13)$; Tomás de Aquino (I, q. 93, a. 6).
6. Una traducción más conforme al original alemán: "El amor creatural completo [la familia natural: padremadre-hijo] es una auténtica imago Trinitatis".

7. El padre Orbe (1964) encontró una larga tradición en la patrística oriental.

8. Un mayor desarrollo puede encontrarse en mi estudio: Cultura, naturaleza y persona: género y estructura personal (Castilla de Cortázar, 2011).

9. San Metodio de Olimpo (El Banquete, III, c. $8,72-73, \mathrm{SCH}$, n. 95 , pp. 108109), en las que denomina al Espíritu "Costa Verbi", costilla del Verbo. 


\section{BIBLIOGRAFÍA}

Arendt, H. (1958/1993). The Human Condition. Chicago: The University of Chicago Press. [Trad. esp. La condición humana. Barcelona: Paidós].

Aristóteles (IVa.C./1994). Reproducción de los animales. Madrid: Gredos.

Børresen, K. E. (1985). Imago Dei, privilège masculin? Interprétation augustinienne et pseudo-augustinienne de Gen 1, 27 y 1 Cor 11, 7. Augustinianum, 25, pp. 213-234.

Børresen, K. E. (1990). Imagine di Dio e modelli di genere nella Tradizione cristiana. En: Spinsanti, S. (coord). Maschio-femmina: dall'uguaglianza alla reciprocitá. Milano: CISF, Ed. Paoline, pp. 113-125.

Børresen, K. E. (1992). Imagen actualizada, tipología anticuada. En: Macciocchi, M. A. Las mujeres según Wojtyla. Madrid: Ediciones Paulinas, pp. 181-195.

Buytendijk, F. J. J. (1966). La mujer: Naturaleza, apariencia, existencia. Madrid: Revista de Occidente.

Castilla de Cortázar, B. (1996). La Trinidad como Familia. Analogía humana de las procesiones divinas. Annales Theologici, 10, pp. 381-416.

Castilla de Cortázar, B. (2005). ¿'Fue creado e varón antesquela mujer? Consideraciones en torno a la antropología de la Creación. Madrid: Rialp.

Castilla de Cortázar, B. (2011). Cultura, naturaleza y persona: género y estructura personal. En: Aparisi, Á. (ed.). Persona y Género. Pamplona: Aranzadi, pp. 423-465.

Castilla de Cortázar, B. (2011). La supuesta pasividad de la mujer. En: Siglo XXI: el siglo de oro de las mujeres. Madrid: Universitas, pp. 14-21. Disponible en http://arvo.net/hombre-mujer/ la-supuesta-pasividad-de-la-mu/gmxniv382-con10600.htm.

Castilla de Cortázar, B. (2014). Condición sexuada y fecundidad, a imagen de Dios. Revista de Actas IV CIRF, 4, pp. 12-24.

Concilio Vaticano II. Constitución Pastoral Gaudium et Spes. Disponible en vatican.va.
Congregación para la Doctrina de la fe (1976/1978). Declaración Inter insigniores. Madrid: BAC.

Congregación para la Doctrina de la fe (2004). Carta sobre la colaboración del hombre y la mujer en la Iglesia y el mundo. Disponible en vatican.va.

Denzinger-Schönmetzer, H. J. D. (1963). En chiridion Symbolorum. Barcelona: Herder.

Escrivá de Balaguer, J. (1968/1998). Conversaciones. Madrid: Rialp.

Gardner, H. (2011) La inteligencia reformulada: las inteligencias múltiples en el siglo XX. Barcelona Paidós.

Gomis Blanco, A. (1991). La biología en el siglo XIX. Madrid: Akal.

Habermas, J. (2002). Die Zukuntf der menschlichen Natur, Suhrkamp Verlag, Frankfurt: Suhrkamp Verlag. [Trad. esp. El futuro de la naturaleza humana. Barcelona: Paidós].

Juan Pablo II (1979). Homilía 28 de enero. Disponible en vatican.va

Juan Pablo II (1981). Exhortación apostólica Familiaris consortio. Disponible en vatican.va

Juan Pablo II (1988). Carta Apostólica Mulieris dignitatem. Disponible en vatican.va

Juan Pablo II (1994). Carta a las familias. Disponible en vatican.va

Juan Pablo II (1995). Carta a las mujeres. Disponible en vatican.va

Juan Pablo II (1995). Encíclica Evangelium vitae. Disponible en vatican.va

Juan Pablo II (2006). Man and woman $\mathrm{He}$ Created Them. A Theology of the Body. Boston: Pauline.

Jung, C. G. (1954/2009). Von den Wurzeln des Bewusstseins. Olten: Walter Verlag. [Trad. esp. Arquetipos e inconsciente colectivo. Barcelona: Paidós].

Ladaria, L. F. (1993). Introducción a la Antropología Teológica. Estella: Verbo Divino.

Marías, J. (1995). Antropología metafísica. Madrid: Alianza.
Orbe, A. (1964). La procesión del Espíritu Santo y el origen de Eva. Gregorianum, 45 , pp. $103-118$.

Platón. (IVa.C./1999). El Banquete. Madrid: Gredos.

Polo, L. (1993). Presente y futuro del hombre. Madrid: Rialp.

Polo, L. (1999/2003). Antropología trascendental (I: La persona humana). Pamplona: Eunsa.

Polo, L. (2008). Planteamiento de la antropología transcendental. En Falgueras, I. y García, J. (coords.). Antropología y transcendencia. Universidad de Málaga, pp. 9-29.

Polo, L. (2011). La esencia del hombre. Pamplona: Eunsa.

Pontificio Consejo "Justicia y Paz». (2005). Compendio de la Doctrina Social de la Iglesia. Madrid: BAC.

Ratzinger, J. (1976). Palabra en la Iglesia. Salamanca: Sígueme

San Agustín. De Trinitate. Madrid: BAC.

Schüssler Fiorenza, E. (1983/1989). In Memory of her. A feminist Theological Reconstruction of Christian origins, New York: Crossroad. [Miguel, P. de (2004). Prácticas de interpretación bíblica. Una guía de lectura de "Pero ella dijo" de E. Schüssler Fiorenza. Bilbao: Desclée de Brower].

Scola, Á. (2001). Hombre-mujer. El misterio nupcial. Madrid: Encuentro.

Scola, Á. (2005). La experiencia humana elemental. La veta profunda del magisterio de Juan Pablo II. Madrid: Encuentro.

Tomás de Aquino (XIII/1959). Summa Theologiae (tercera parte). Madrid: BAC.

Von Balthasar, H. U. (1994). Estados de la vida del cristiano. Madrid: Encuentro.

Von Balthasar, H. U. (1998). Teologica, III. Madrid: Encuentro.

Weiger, G. (1999). Biografía de Juan Pablo II. Testigo de esperanza. Madrid: Plaza Janés.

Wojtyla, K. (1982). Persona y acción. Madrid: BAC. 